2020-03-13

\title{
The impact of a spatial occlusion training intervention on pass accuracy across a continuum of representative experimental design in football
}

\author{
Alan Dunton \\ Technological University Dublin, alan.dunton@tudublin.ie \\ Cian O' Neill PhD \\ Munster Technological University, cian.oneill@mtu.ie \\ Edward K. Coughlan PhD \\ Munster Technological University, edward.coughlan@mtu.ie
}

Follow this and additional works at: https://arrow.tudublin.ie/tfschhmtart

Part of the Sports Studies Commons

\section{Recommended Citation}

Alan Dunton, Cian O'Neill \& Edward K. Coughlan (2020) The impact of a spatial occlusion training intervention on pass accuracy across a continuum of representative experimental design in football, Science and Medicine in Football, 4:4, 269-277, DOI: 10.1080/24733938.2020.1745263

This Article is brought to you for free and open access by the School of Tourism \& Hospitality Management at ARROW@TU Dublin. It has been accepted for inclusion in Articles by an authorized administrator of ARROW@TU Dublin. For more information, please contact arrow.admin@tudublin.ie, aisling.coyne@tudublin.ie, gerard.connolly@tudublin.ie.

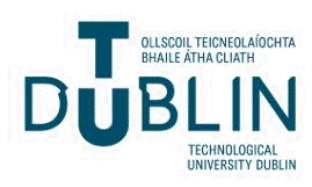




\title{
The impact of a spatial occlusion training intervention on pass accuracy across a continuum of representative experimental design in football
}

\author{
Alan Dunton, Cian O'Neill and Edward K. Coughlan \\ Department of Sport, Leisure \& Childhood Studies, Cork Institute of Technology, Cork, Ireland
}

\begin{abstract}
Introduction: The ability to successfully complete a pass in football can positively impact the result of the game. While previous work has identified the importance of perceptual behaviours before and during passing action, there is a paucity of research analysing the impact of training interventions on pass performance.

Methods: A tri-phasic approach was employed to assess the impact of training with spatial occlusion goggles. Each phase was designed to assess participants' ability to control and pass a football during a representative experimental task. The study design consisted of a pre-test, 2-week training intervention, post-test and 2-week retention test.

Results: Significant improvements in response accuracy $(p<.05)$ and response time $(p<.05)$ were displayed across all three phases for those who wore occlusion goggles. Control error $(p<.05)$ showed a significant improvement during phase one and phase two only. There were no sustained significant changes for those who did not wear the occlusion googles.

Conclusion: Findings suggest that guiding the visual system away from the lower limbs while receiving a football and towards relevant information, the movement of a particular participant, within the environment can improve pass accuracy and speed of pass following a training intervention with occlusion goggles.
\end{abstract}

ARTICLE HISTORY

Accepted 13 March 2020

\section{KEYWORDS}

Spatial occlusion; football; training intervention; skill acquisition; representative experimental design

\section{Introduction}

Effective passing metrics such as total passes, average pass streak and pass success rate have been shown to positively influence overall team performance and the outcome of a game in football (Bush et al. 2015; Liu, Gomez, Lago-Penas, \& Sampaio, 2015). While the research of Bush et al. (2015) and Liu et al. (2015) provide information regarding the importance of accurate and effective passing in football, it does not provide information relating to the perceptual-cognitive attributes of effective passing. Research analysing perceptual skills such as scanning, before and during possession of the football, has been conducted in both a laboratory setting (McGuckian et al. 2018a) and in-situ during 11v11 match play (McGuckian et al. 2018b). Both studies identified a relationship between an increase in head turn frequency prior to receiving the football and the speed and direction of the passes. Furthermore, it was demonstrated that during the time periods leading to attaining possession, higher head turn frequency and larger excursions were prevalent, suggesting that as players recognised that they were due to receive the ball, they engaged in more exploratory behaviour (McGuckian et al. 2018b).

Although research has identified the importance of perceptual skills before passing, and passing on overall performance in football (Bush et al. 2015; Liu et al. 2015; McGuckian et al. 2018a, $2018 \mathrm{~b})$, research examining the impact of perceptual training to improve passing actions is limited. A method often used to train perceptual ability is visual occlusion, which can be sub- categorised as spatial or temporal occlusion. Spatial Occlusion has traditionally been identified as the process of masking or removing information sources from video-based film clips. Temporal Occlusion refers to the process of removing or masking visual information across different time periods (Vickers 2007). Spatial and temporal occlusion research initially focused on identifying differences in performance variables, such as gaze behaviour and anticipation, of expert and novice athletes (Jones and Miles 1978; Abernethy 1990). However, a number or researchers have since used visual occlusion, predominantly a temporal paradigm, as part of a training intervention in football. The majority of this research focused on the effectiveness of perceptual training with goalkeepers (Poulter et al. 2005; Murgia et al. 2014). Participating cohorts in these studies were assessed for their ability to anticipate penalty kick destinations at pre- and post-tests through a video-based temporal occlusion paradigm. Murgia et al. (2014) utilised a temporal occlusion paradigm that occluded the video of a penalty kick at football contact during testing phases. The training intervention required participants to identify the destination of penalty kicks on a computer screen by clicking on one of four white squares containing a question mark. Poulter et al. (2005) temporally occluded penalty kick videos one frame $(0-40 \mathrm{~ms})$ prior to football contact. Participants were provided with explicit or implicit instruction after each trial during the training intervention, depending on which group they were assigned to, as they verbally responded to temporal occlusion video clips. 
A more recent study focused on the impact of video-based temporal occlusion on decision-making and reactive agility in football (Nimmerichter et al. 2015). During the pre-test, posttest and training intervention, participants were required to respond to video clips that displayed four different defensive tackles. Participants were required to mark 'left' or 'right' on a form to identify which direction they thought the attacker would successfully go by the defender. Findings demonstrated significant improvement in response time and response accuracy for those who underwent temporal occlusion training. Nimmerichter et al. (2015) also implemented a transfer test (see Sheppard et al. 2006, for details of assessment), to assess participants' ability to respond to the movement of a human stimulus by running left or right. Results of the transfer test displayed a significant improvement in sprint times for those who took part in temporal occlusion training, which highlights the potential transfer toward game-play.

It is important to note the dominance of the temporal occlusion paradigm in the research presented above and distinct lack of spatial occlusion research. A limitation often associated with temporal occlusion is that the entire visual field is occluded at a particular time point (Williams and Jackson 2019). This means that the visual system is not being directed toward a particular source of information that may guide actions. This limitation highlights the need for spatial occlusion research to become more prominent within the domain. A further limitation of temporal occlusion-based research is the reliance on video simulations and response methods, such as verbal and written responses, which decouple perception-action. Perceptionaction coupling can be identified as the cyclical relationship between the perception of information afforded by the environment and the specific actions that emerge as a result of what is perceived, (Vickers 2007). The importance of maintaining perception-action coupling has been exhibited in research assessing expert prediction in tennis (Farrow and Abernethy 2003), where findings demonstrated superior results for a tennis task performed with a coupled response rather than an uncoupled response. Further research to display the importance of representative design and perception-action coupling was conducted by Dicks et al. (2010), who compared goalkeepers gaze behaviour and performance during penalty kicks under varied conditions. Participants were required to; verbally respond to a video simulation, respond to a video simulation with a movement, verbally respond in situ, respond in situ with a directional movement and respond in situ with an interception. Findings demonstrated higher save rates when participants were required to intercept the football in-situ. In addition to this gaze behaviour was fixated earlier and for longer on ball location. This differs to the video simulation conditions where initial gaze behaviours were directed toward the head or torso and subsequently toward the kicking and non-kicking legs.

A framework which supports the findings of Dicks et al. (2010), that was introduced by Van der Kamp et al. (2008), heavily influenced by Gibson (1979), further emphasises the importance of perception-action coupling. The framework outlines the significance of the two-visual system model by Milner and Goodale (1995) for anticipation. This two-visual system model defines the role of the dorsal system's vision for action, and ventral system's vision for perception, and the importance of both systems being engaged during performance. If experimental designs remove the need to perform the action associated with what is perceived, such as utilising a verbal or written response to a pass in football, the ventral system is dominant while the role of the dorsal system, which is primarily used during control and passing actions in football, is limited. With reference to football, players often utilise perceptual information such as postural cues from a teammate and the movement of the football to identify the destination of a pass. Additional information such as the position of opposing players and the directional movement of a teammate must also be obtained in order to play a successful return pass. The adequate sampling of such information, during training, is essential for maintaining the functional coupling of perception-action processes in representative environments (Pinder et al. 2011). The conditions of a representative experimental environment must represent the behavioural setting they are intended to be applied to (Araújo et al. 2007). Therefore, the preservation of perception-action coupling in experimental research where participants must identify information from a teammate and perform the requisite task of passing a football based on the subsequent movement of the teammate can further our understanding of how humans interact within the environment and improve the transfer of skill to the sporting domain (Dicks et al. 2009).

In order to conduct perceptual training during a more representative experimental design and address the potential issues often associated with video-based occlusion, tools such as spatial occlusion goggles (see Figure 1) can be utilised. Spatial occlusion goggles eliminate the vision of the low-grade visual field from up to 2.6 metres when the participants head is in a neutral, straightforward position and attempting to gaze down, including the lower limbs and the football during the latter stage of a pass. To date, two studies have been conducted using this particular form of spatial occlusion in basketball (Dunton, O'Neill, Jermyn, Dawson, \& Coughlan, 2019a) and football (Dunton, O'Neill, \& Coughlan, 2019b). The latter assessed the impact of spatial occlusion on receiving and passing a football while concurrently calling a series of randomly generated numbers placed in front of participants. Results demonstrated that those in the spatial occlusion group

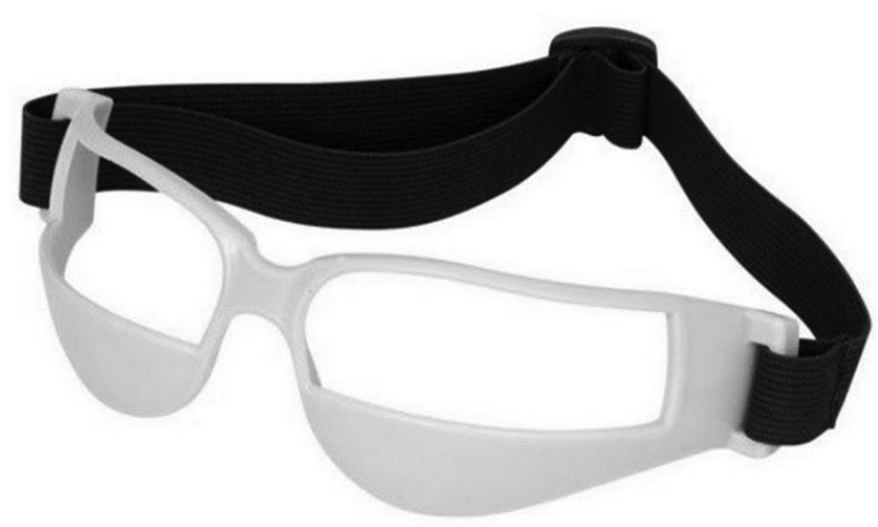

Figure 1. Spatial Occlusion Goggles used by participants in the OCC Group. 


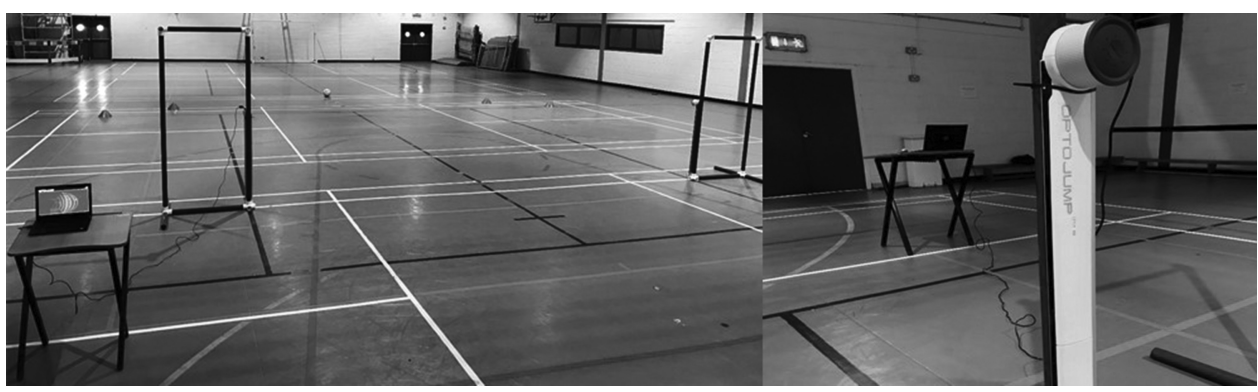

Figure 2. OptoJump timing gate system secured vertically and view of experimental design.

significantly decreased number call error and pass accuracy error from pre-test to post-test and also at the 2-day retention test. It warrants a mention that participants were required to respond to a pace-controlled ball feeding machine and a visual stimulus that are not a direct representation of the game environment. These limitations, which appear frequently throughout visual occlusion literature, can be addressed by implementing spatial occlusion goggles, in situ while maintain perception-action coupling with football relevant stimuli.

A key determining factor of successful passing performance is the use of perceptual information from teammate's, such as postural cues, opposition players and the movement of the football during play. After a football has been passed, the movement of the ball can be predictable, so the most useful information for a player to complete a subsequent pass is that which relates to the movement of their teammates or opponents and less so the football (Oppici et al. 2017). Therefore, it may be beneficial for a player to learn to use the perceptual information from these relevant sources in order to improve performance. To achieve this, using spatial occlusion googles in training may guide players toward this useful information by removing the ability to use perceptual information from the football. This is likely to result in improved performance once players have learned to make use of the more useful information once the occlusion goggles are removed. Therefore, the purpose of the current study was to assess the impact of spatial occlusion goggles on pass accuracy, speed of pass, and ball control, during an experimental design more representative of the game of football than previous research in the domain (Dunton et al. 2019b; Poulter et al. 2005; Murgia et al. 2014; Nimmerichter et al. 2015). It was expected that participants who completed the training intervention phase with the spatial occlusion goggles would experience a reduction in response time (RT), an improvement in response accuracy (RA) and a reduction in control error (CE) once full visual conditions were returned.

\section{Methodology}

\section{Participants}

Seventy-two participants were recruited for this study across three phases. Thirty, third-level sports management students ( $M=19.3$ years old, $S D=2.3$ ), who had a minimum of three years playing experience in competitive football, participated in phase one and were randomly assigned to one of three groups;
Occlusion (OCC), Practice (PRA) and Control (CON). A further thirty sports management students $(M=20.5$ years old, $S D=1.9$ ), with a minimum of three years playing experience in competitive football, participated in phase two and were randomly assigned to one of three groups; Occlusion (OCC), Practice (PRA) and Control (CON). Finally, twelve skilled male football players $(M=21.1$ years old, $S D=3.5)$, with a minimum of 10 years playing experience, were selected for phase three as a single intervention group, Occlusion (OCC). Each participant had normal or corrected-to-normal vision. Ethical approval was attained from the host institution's research ethics committee.

\section{Experimental set-up, materials, and apparatus}

The experimental set-up covered a $14 \mathrm{~m} \times 9 \mathrm{~m}$ surface area beginning with the researcher seated at the rear left zone of the testing area, with a Dell Latitude 7290 Laptop, $2 \mathrm{~m}$ behind the start line for the participant being assessed. Two 'goals', comprised of two cones, located $8 \mathrm{~m}$ from the Microgate Optojump system and $8 \mathrm{~m}$ apart from one another were set $1.5 \mathrm{~m}$ apart and angled at 70 degrees toward the participant's start position. The start line, where the football was set to be passed from, was positioned $10 \mathrm{~m}$ from the Optojump gate. A Sony HDR video camera was located at the back of the testing area to record testing sessions.

The Microgate Optojump sensors were secured, via cable ties, to a custom built frame and hung vertically $6 \mathrm{~m}$ apart and $1 \mathrm{~m}$ in front of the participant's starting position (Figure 2). The Optojump software was set to record the time lapse of a football (White Nike Size 5) passing through the Optojump sensors in milliseconds. Phase one and two were conducted in an indoor sports hall, and phase three was conducted on a football pitch.

\section{Procedure}

A representative football task was designed to analyse the impact of a training intervention, using spatial occlusion goggles (Dunton et al., 2019b), on controlling and passing a football across three separate phases (Figure $3(a-c)$ ). All participants were required to complete a pre-test, 2-week training intervention, post-test, and 2-week retention test and instructed to arrive at the testing and intervention phases in appropriate training attire. A participant information sheet, outlining the study in detail, and a participant consent form were provided to each participant upon arrival. Subsequent to 


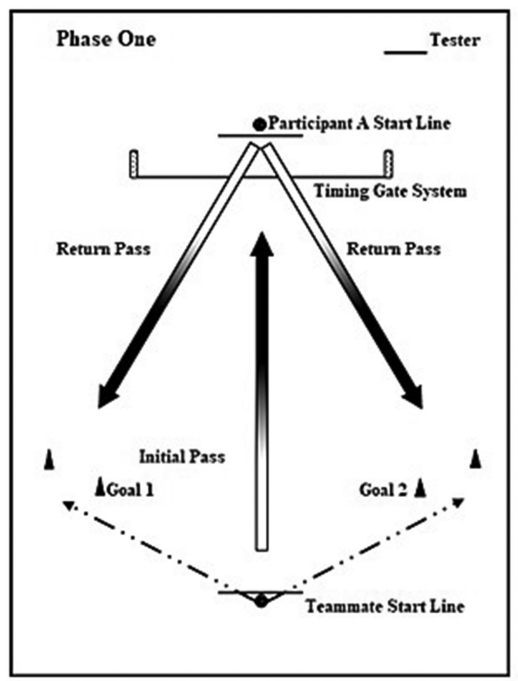

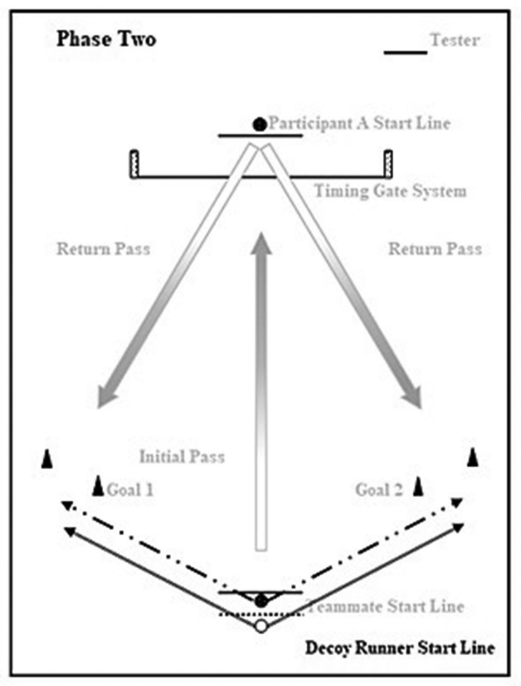

b

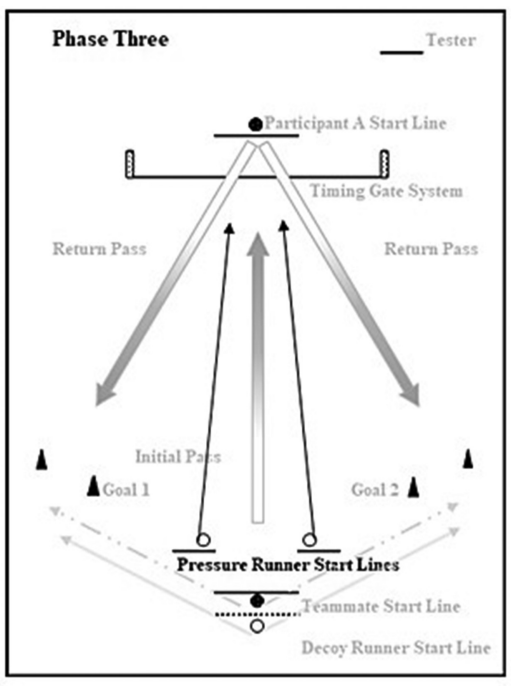

c

Figure 3. $(a-c)$ Scaled view of the experimental design for each phase.

participants filling out the participant consent form a number of scripted instructions were explained to each participant outlining the testing and training intervention in detail.

\section{Phase one and two - testing}

Testing phase one (Figure 3(a)) consisted of a 1-to-1 passing task which began with the participant being assessed (Participant A) situated at the designated start line behind the Optojump timing system. This start line was the reset position for Participant A for each trial. A 'teammate' was located $11 \mathrm{~m}$ away with the football. For the purpose of this manuscript the participant who played the initial pass in each trial will be referred to as 'teammate'. The teammate was instructed to provide a firm pass of the football along the floor to Participant A. After completing the pass the teammate was instructed to run to their left or right as indicated by the Tester, who was situated behind Participant $A$, to receive the return pass. If the tester deemed the initial pass to be unacceptable, i.e., the ball travelled through the air or was passed too far to the left or right of the participant, the trial was dismissed and participants were instructed to reset and repeat the trial. Participant A was instructed to take one touch to control the football before passing it back to the teammate through the designated 'goal' as fast as possible. It was emphasised that the goal was the primary target for the return pass. If the teammate stopped before or after the goal participants were to still aim for the goal regardless. Participant $A$ was informed that taking more than one touch to control the football or passing the football back with their first touch would result in a control error. Participant $A$ was also informed that if the football travelled forward by $1 \mathrm{~m}$ before the return pass it would also result in a control error. Every four trials the teammate was replaced in order to prevent fatigue and prevent participants from becoming familiar with the passing behaviours of a single teammate. Each participant had a minimum of four separate teammates and completed 20 trials in each testing phase.

Phase Two (Figure 3(b)) progressed to include an additional participant, a 'decoy runner', to increase passing task difficulty. The decoy runner was instructed to stand $0.5 \mathrm{~m}$ behind the teammate at the beginning of each trial. Once the football was passed, the decoy runner was instructed to run in the opposite direction of the teammate. As in phase one both the teammate and decoy runner were replaced every four trials. All other elements of the test remained identical to phase one.

\section{Phase one and two - training intervention}

The training intervention for phase one and two required participants to replicate the passing task performed in the testing phases respectively. Each pass performed in the training intervention was identical to a trial in the testing phase. Participants in the OCC groups completed a two-week, four session, training intervention consisting of 60 passes per session with the spatial occlusion goggles on. The 60 passes were divided in to four blocks of 15 passes. Participants in the PRA groups completed the same intervention as the OCC groups without the spatial occlusion goggles and those in the CON group completed testing protocols only.

\section{Phase three - testing}

Testing in phase three (Figure 3(c)) consisted of a 2-vs-2 passing task which introduced an additional layer of complexity from phase two with the introduction of a 'pressure runner'. The pressure runner was instructed to begin $1 \mathrm{~m}$ in front and to the left or right hand side of the teammate. The start position for the pressure runner was randomly organised for each trial. Once the initial pass was played to Participant $A$, the pressure runner was instructed to run toward Participant $A$ but not to actively tackle. As in phase one and two, the teammate, decoy runner and pressure runner were replaced every four trials. All 


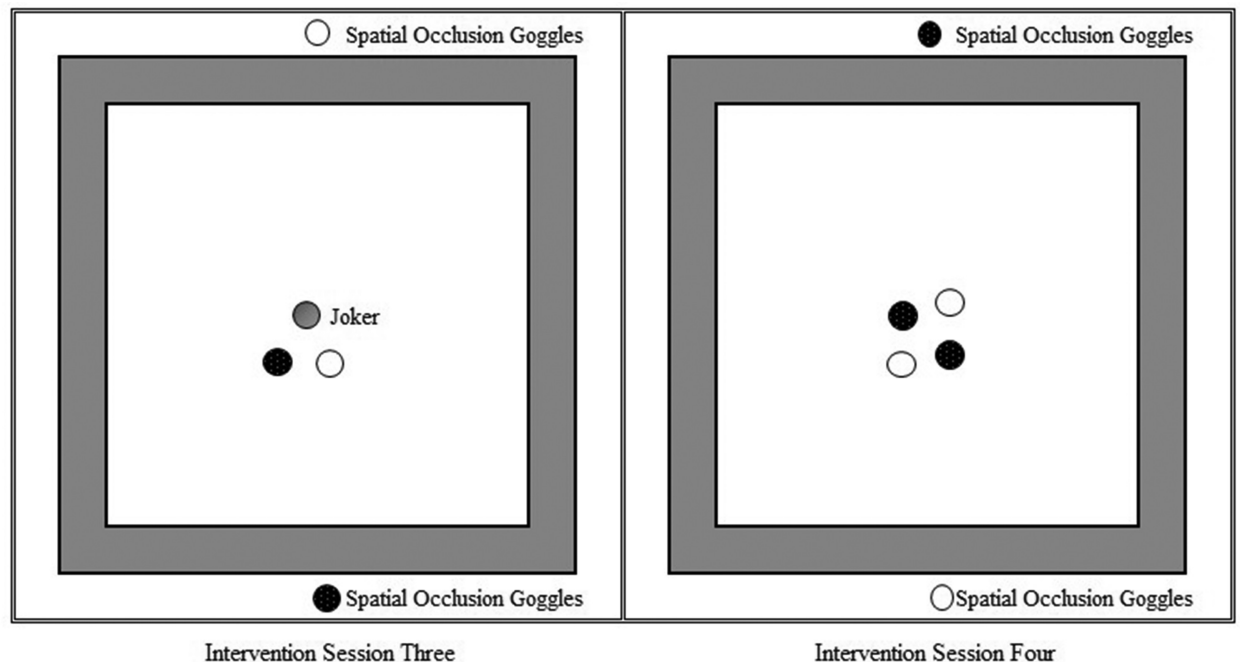

Figure 4. Scaled view of the phase three training intervention, session three and session four.

other elements of the testing phases remained identical to phase one and two.

\section{Phase three - training intervention}

The training intervention for phase three, specifically designed for the current study, was intended to represent standard training tasks conducted in an elite football setting and therefore was not conducted in the same manner as phase one and two. The purpose of this design modification was to maximise the translation impact of the current research. All participants in phase three wore the spatial occlusion goggles for the duration of the twoweek, four session, training intervention. Session one and two were identical to the training interventions conducted by participants in phase one and two respectively. Session three and four (Figure 4) were conducted using possession games. Session three had two participants wearing the spatial occlusion goggles on the periphery of an $11 \mathrm{~m} \times 11 \mathrm{~m}$ grid. An inner $(10 \mathrm{~m} \times 10 \mathrm{~m})$ grid contained three players, with one player assigned as a teammate to each participant wearing the occlusion goggles on the periphery. The third player within the grid was instructed to act as a 'floating teammate' to the team in possession of the football. Participants wore the occlusion goggles for one and a half minutes before rest interval of 30 seconds, which was repeated four times. Session four was also conducted using a possession game; again two participants wore the occlusion goggles situated on the outside of the grid. However, during this session, four players were situated in the inner grid with two players assigned to each participant on the periphery to create a 3-vs-3 possession game. Those wearing the occlusion goggles on the periphery were a minimum of $1 \mathrm{~m}$ away from the players in the inner $10 \mathrm{~m} \times 10 \mathrm{~m}$ grid. The role of those wearing the occlusion goggles was to use only one touch to control the football before playing a return pass to a teammate inside the grid. This was designed to remain consistent with the protocol used during the respective testing phases. As in session three, the occlusion goggles were worn for one and a half minutes before rest interval of 30 seconds, which was repeated four times.

\section{Statistical analysis}

Three variables were selected for analysis; Response Time (RT), Response Accuracy (RA), and Control Error (CE). RT, measured via the Optojump, was defined as the duration of time in milliseconds, from the moment the football passed through the timing gate system to the moment it passed back through from the return pass. RA were recorded as a successful pass of the football through the correct goal, which was identified by the directional run of the teammate. CE was recorded when participant's (i) needed more than one touch to control the football, (ii) allowed the football to travel forward by $1 \mathrm{~m}$ before providing the return pass, or (iii) passed the football back with their first touch.

Statistical analyses were conducted using the IBM SPSS statistics package (version 25). A 3-group x 3-test mixed between-within MANOVA was conducted to analyse the impact of the experimental intervention on all performance variables for phase one and phase two. However, due to the change in phase three only having one experimental group a 1-group $x$ 3-test repeated measure MANOVA was conducted. An assessment for normality and relevant assumptions were investigated at each stage of the analysis for phase one, two and three. Post Hoc tests, using a Bonferroni correction, were also conducted with syntax applied to provide pairwise comparisons. The alpha level required for significance was set at $p<0.05$ with the confidence interval set at $95 \%$. Partial eta squared was utilised to assess effect size. Effect size can be interpreted using guidelines proposed by Cohen (1988), $.01=$ small effect, $.06=$ moderate effect, .14 = large effect.

\section{Results}

\section{Phase one}

An analysis of the results of the 3-group $x$ 3-test mixed betweenwithin repeated measures MANOVA revealed a significant interaction effect $F(12,44)=6.29, p<.001, \eta_{p}^{2}=.632$. 


\section{Response accuracy}

Results of the repeated measure MANOVA revealed no significant mean difference between groups at pre-test $(p>.05)$. There was a significant increase in RA from pre-test $(M=54 \%$, $S D=7 \%)$ to post test $(M=73 \%, S D=4.8 \%), p<.05,95 \% \mathrm{Cl}$ $[-4.86,-2.74]$ and retention test $(M=71.5 \%, S D=4.7 \%)$, $p<.05,95 \% \mathrm{Cl}[-4.54,-2.45]$ for the OCC group (Figure 5(a)) with no significant change from post-test to retention test. The PRA group also displayed a significant increase from pre-test $(M=55 \%, S D=7.8 \%)$ to post test $(M=60.5 \%, S D=4.4 \%)$, $p<.05,95 \% \mathrm{Cl}[-2.16,-.04]$. However, this was not maintained at the retention test $(M=53.5 \%, S D=8.5 \%)$, with a significant decrease in RA demonstrated ( $p<.05,95 \% \mathrm{Cl}[-.75,1.34])$. There was no significant change across any test for the CON group.

\section{Response time}

A significant decrease in RT was recorded from pre-test $(M=0.983 \mathrm{~ms}, S D=0.128)$ to post-test $(M=0.924 \mathrm{~ms}$, $S D=0.062), p<.05,95 \% \mathrm{Cl}[.01, .12]$ for the OCC group (Figure 5(b)). However, no significant change was found from pre-test to retention test $(M=0.936 \mathrm{~ms}, S D=0.046)$, ns (nonsignificant) $p>.05,95 \% \mathrm{Cl}[-.02, .12]$. There was no significant change experienced across any test for the PRA or CON group.

\section{Control error}

The OCC group experienced a significant decrease $(p<.05,95 \%$ $\mathrm{Cl}[1.96,3.44])$ in $\mathrm{CE}$ from pre-test $(M=18.5 \%, S D=5.8 \%)$ to posttest $(M=5 \%, S D=3.3 \%)$ and retention test $(M=3.5 \%$, $S D=4.1 \%), p<.05,95 \% \mathrm{Cl}[2.29,3.70]$. There was no significant difference between the post and retention test (ns, 95\% $\mathrm{Cl}[-.15, .75])$. A significant decrease in $\mathrm{CE}$ was experienced for the CON group from pre-test $(M=11 \%, S D=9.4 \%)$ to post-test $(M=6 \%, S D=8.4 \%), p<0.05,95 \% \mathrm{Cl}[.26,1.73])$. The result for pre-test to retention test $(M=7.5, S D=10.6 \%)$ was reported as $p=.051,95 \% \mathrm{Cl}[-.01,1.40]$. While this result is not significantly significant, is important to note that it was .001 away from statistical significance (Figure 5(c)). There were no significant improvements experienced by the PRA group across any test.

\section{Phase two}

A significant interaction effect was reported from the results of the repeated measures MANOVA $F(12,44)=6.97, p<.001$, $\eta_{p}^{2}=.655$.

\section{Response accuracy}

Results of the repeated measure MANOVA for phase two displayed a significant increase in RA for the OCC group (Figure 6(a)), $\mathrm{p}<.05$, $95 \% \mathrm{Cl}[-5.18,-3.22]$ from pre-test $(M=57 \%, S D=11.1 \%)$ to post $(M=78 \%, S D=6.3 \%)$ and retention test $(M=74 \%, S D=5.7 \%)$, $p<.05,95 \% \mathrm{Cl}[-4.37,-2.43]$. No significant change was evident from post-test to retention test (ns, 95\% Cl [-.09, 1.69]). There was no significant change in RA experienced by the PRA or CON groups across any test. Pairwise comparisons also displayed no significant difference in RA between any groups at pre-test.

\section{Response time}

A significant decrease in RT (Figure 6(b)) for the OCC group was revealed from pre- $(M=1.001 \mathrm{~ms}, S D=.093)$ to post-test $(M=.914 \mathrm{~ms}, S D=.058) p<.05,95 \% \mathrm{Cl}[.05, .12]$ and retention test $(M=.917 \mathrm{~ms}, S D=.049), p<.05,95 \% \mathrm{Cl}[.04, .12]$. There was no significant change from post-test to retention test $(p>.05$, $95 \% \mathrm{Cl}[-.03, .02])$. With the exception of a significant increase

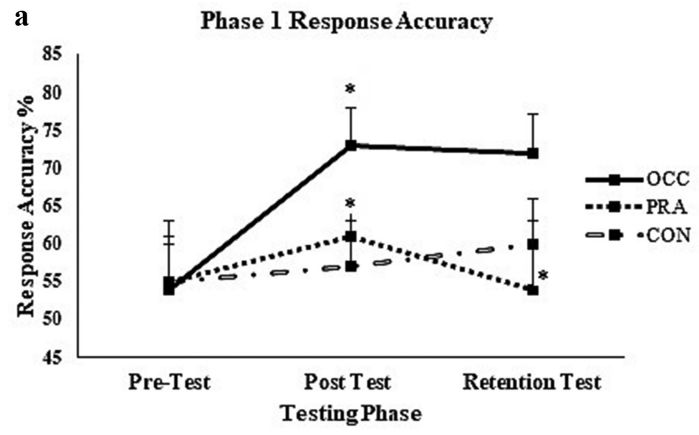

b

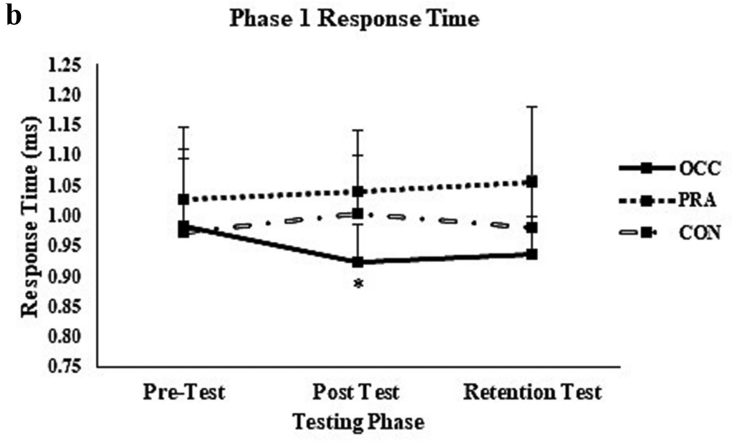

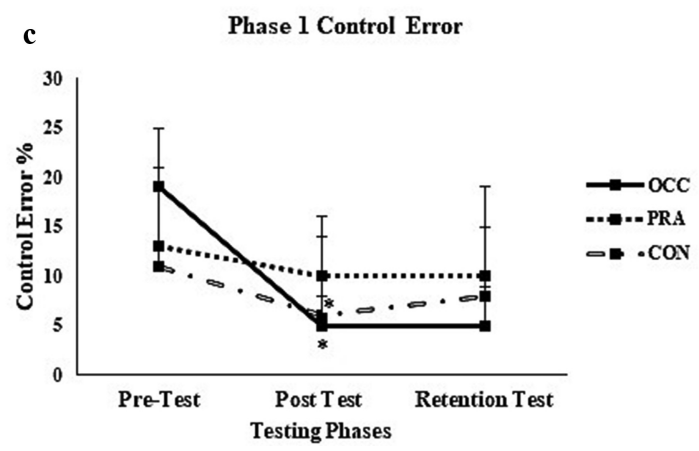

Figure 5. (a) Mean scores for response accuracy for each group during phase 1, (b) Mean scores for response time for each group during phase 1, (c) Mean scores for control error for each group during phase 1 , error bars indicate standard deviation with ${ }^{* \prime \prime}$ used to identify significant changes. 


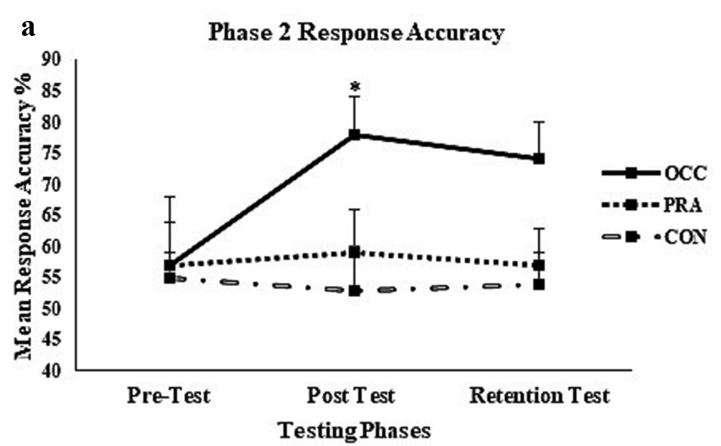

Phase 2 Response Time
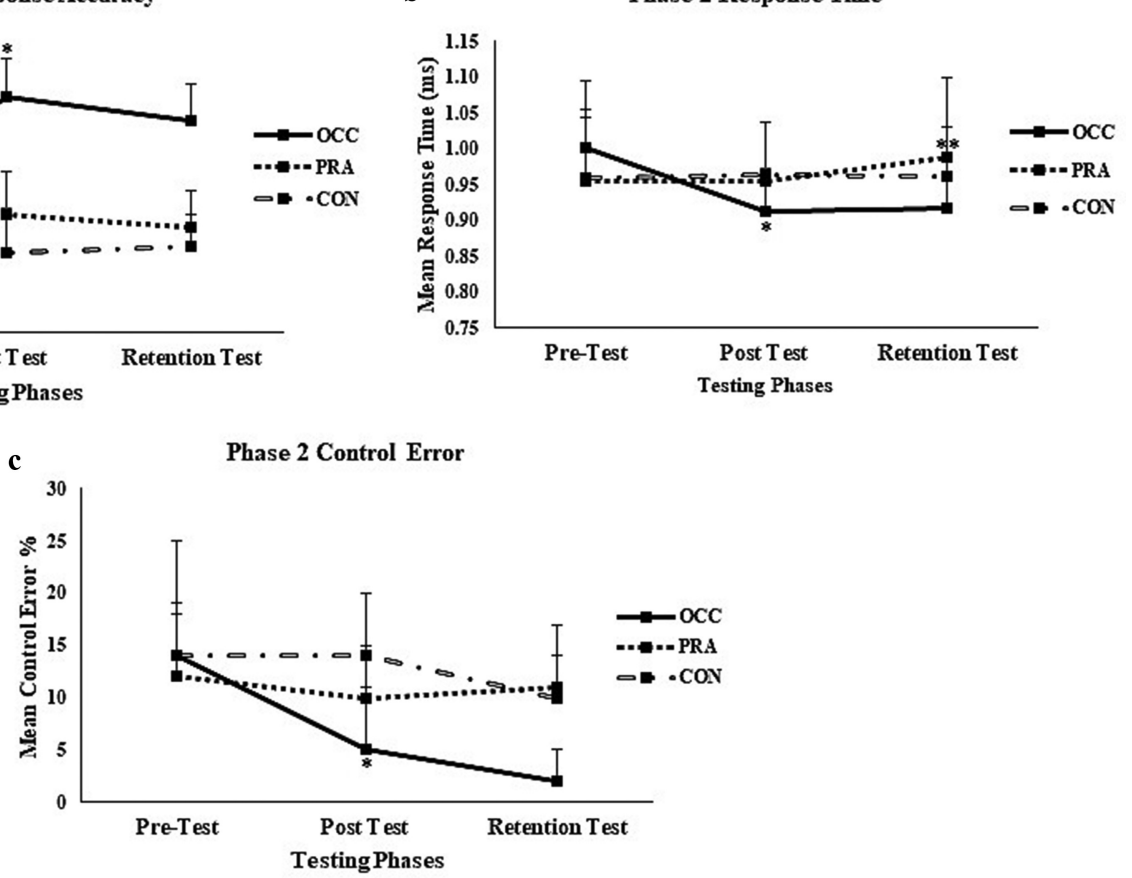

Figure 6. (a) Mean scores for response accuracy for each group during phase 2, (b) Mean scores for response time for each group during phase 2, (c) Mean scores for control error for each group during phase 2, error bars indicate standard deviation with "*' used to identify significant changes.

in RT from post-test to retention test $(p<.05,95 \% \mathrm{Cl}[-.06$, $-.01]$ ) for the PRA group, there was no other significant change in RT for the PRA or CON group.

\section{Control error}

A significant decrease $p<.05,95 \% \mathrm{Cl}[.91,2.88]$ was displayed in CE for the OCC group from pre- $(M=14 \%, S D=10.7 \%)$ to post-test $(M=4.5 \%, S D=6 \%)$ and retention test $(M=2 \%$, $S D=3.5 \%), p<.05,95 \% \mathrm{Cl}[1.39,3.41]$. There was no significant difference between the post and retention test (ns, 95\% Cl $[-.19,1.20])$. There were no significant changes reported for the PRA or CON group across any test (Figure 6(c)).

\section{Phase three}

An analysis of the results of the 1-group $\times 3$-test MANOVA displayed a significant main effect $F(6,6)=56.98, p<.001, \eta_{p}^{2}=.983$.

\section{Response accuracy}

Results of the MONOVA displayed a significant improvement from pre- $(M=65 \%, S D=7 \%)$ to post-test $(M=83.3 \%$, $S D=6.2 \%), p<.05,95 \% \mathrm{Cl}[-4.39,-2.94]$ and retention test $(M=81.3 \%, S D=4.8 \%), p<.05,95 \% \mathrm{Cl}[-4.29,-2.20]$. There was no significant difference reported from post-test to retention test (ns, 95\% Cl $[-.39,1.23])$.

\section{Response time}

Significant decreases in response time were experienced from pre- $(M=1.089 \mathrm{~ms}, S D=.061)$ to post-test $(M=.963 \mathrm{~ms}$, $S D=.048), p<.05,95 \% \mathrm{Cl}[.09, .16]$ and retention test $(M=.970 \mathrm{~ms}, S D=.042), p<.05,95 \% \mathrm{Cl}[.08, .15]$. Analysis of the post-test to retention test displayed no significant change (ns, 95\% Cl $[-.02, .01]$ ).

\section{Control error}

There was no significant change in Control Error across any test $(p>.05)$.

\section{Discussion}

The purpose of the current research was to assess the impact of spatial occlusion goggles on response accuracy, response time and control error during representative experimental tasks. Analysis of results from phase one displayed some findings that were not expected by the authors. Primarily, there was a significant improvement for the PRA group in RA from pre- to post-test. However, the significant improvement was not maintained at the retention test. This would suggest that any improvement experienced was as a result of a practice effect or familiarity with the test. Additionally, a significant decrease in CE was experienced for the CON group. However, the decrease may have occurred as a result of a trade off in RT, as participants performed the task slower, which increased from pre-test $(M=.972, S D=.123)$ to post-test $(M=1.05, S D=.094)$ and retention test $(M=.991, S D=.085)$.

Results for the OCC group demonstrated a significant increase in RA from pre- to post-test and retention test. This group also significantly decreased RT and CE from pre- to posttest with CE also significantly decreasing from pre-test to retention test. However, the significant change for RT was not maintained at the retention test. Results obtained in phase one display similar trends to that of Dunton et al. (2019b) who also assessed the impact of spatial occlusion on receiving and passing a football in a more controlled setting. In both studies, participants significantly improved and retained passing accuracy as a result of the respective training intervention. The significant decrease in number call error displayed by Dunton 
et al. (2019b) suggested an improved ability to guide visual attention toward a particular visual stimulus within the environment. A comparable assessment can be made with the result of the current research as participants significantly improved across each performance variable. These improvements may be attributed to a more efficient identification of the directional run of the teammate, thereby enabling participants to make a faster, more accurate pass. This study shares a similar methodological approach to Dunton et al. (2019b), with the current study placing an emphasis on a representative experimental design. It is important to note this sustained trend in improvement for the spatial occlusion goggles during a more representative experimental task, particularly with the introduction of a human stimulus. Improvements in response accuracy and response time while interacting with a human stimulus suggest the benefits of integrating spatial occlusion goggles in to sport specific training.

Phase two produced a similar trend of results to that of phase one for the OCC group, with significant improvements evident from pre- to post-test and retention test for each performance variable. There were also no significant changes from post-test to retention test in each case, thus demonstrating a learning effect. These findings contrast those of Poulter et al. (2005), who displayed confounding results, with a placebo effect providing significant improvements similar to the implicit and explicit groups, following a perceptual training intervention. In spite of this, methodological concerns must be addressed when comparing the findings of Poulter et al. (2005) to the current research as the former only used a one-day training intervention, which may have been too short a stimulus.

Conversely, the results of the current research complement those of Murgia et al. (2014) and Nimmerichter et al. (2015) who demonstrated significant improvements in performance variables such as response accuracy (Murgia et al. 2014) and response time (Nimmerichter et al. 2015) following a perceptual training intervention. While there are a number of positive comparable results experienced, it is important to note the use of verbal (Poulter et al. 2005; Murgia et al. 2014), and button press with verbal (Nimmerichter et al. 2015) response methods at preand post-test. These response methods decouple perception and action, which may interrupt the underpinning mechanisms needed to perform the sport specific action required. The results of the transfer test from the research of Nimmerichter et al. (2015) provides the most pertinent comparison to the current research as in both instances participants were required to physically respond to the directional movement of a human stimulus. While the current study has taken a positive step forward to examine occlusion via a representative design, the authors acknowledge a number of limitations that need to be addressed in future research. These include in-game variables, such as continued play in a 360 degree environment, additional defenders and teammates impacting passing decisions, and the instruction provided for the return pass potentially shaping behaviours in the current research. However, during gameplay in football and more appropriately training, there is an extensive occurrence of one-to-one, two-on-one, two-on-two, three-ontwo and three-on-three situations. In addition to this, findings from Dicks et al. (2010) display significant differences in gaze behaviour and improvements in performance outcomes when participants were required to intercept a penalty kick in situ rather than providing a verbal or movement response in situ or to a video-simulation. These findings highlight the importance of maintaining the perception-action cycle in the current research with participants required to control the football and return the pass based on the behaviours of the teammate.

The results of the retention tests in the current research are critical to providing a deeper understanding of the impact of the occlusion goggles from a practice and learning effect perspective. While positive findings were recorded during the respective retention tests (i.e., Phase one, two and three), none of the video-based football studies discussed (Poulter et al. 2005; Murgia et al. 2014; Nimmerichter et al. 2015) implemented a retention test. This oversight must be addressed in future research, particularly when the implications of retention tests on motor learning are considered (Magill 2011).

Analysis of findings from phase three remain consistent with that of phase one and two with significant improvements in performance for RT and RA occurring from pre- to post-test and retention test. While there was no significant change in CE evident during this phase, it is imperative to note the very low percentage error recorded across testing phases as a key factor. A mean error of $4.17 \%$ at pre-test equates to a mean of one control error per 20 trials. In addition to this, CE scores decreased at post-test, with a mean error of $1.25 \%$ that equates to a mean of less than one control error per 20 trials, provides an acceptable explanation for the lack of a significant change. The improvements experienced in pass accuracy and time of pass suggest an improvement in efficiency for the performance of the task. As participants in phase three were required to account for the movement of a teammate, decoy runner and pressure runner, it could be postulated that improvements in scanning behaviour were present. Such improvements may be attributable to the spatial occlusion goggles guiding visual attention outward toward the training (game) environment, thus reducing the allocation of attention toward the performance of the skill being performed. This may suggest participants utilised prospective control during the reception of the football where the perceptual information obtained during the sequence may have been used online for movement regulation, a strategy used by ice-hockey goaltenders during the interception of ice-hockey shots in situ (Panchuk and Vickers 2009). This concept is supported by the research of McGuckian et al. (2018a) and McGuckian et al. (2018b), which identified a correlation between higher head turn frequency before receiving the football and the outcome of the pass as well as speed of the pass. The positive findings of phase three need to be tempered with the limitation of a single group experiment design. However, it is important to recognise determining factors while having access to a skilled cohort of players' in-season (Müller et al. 2015).

The positive findings displayed in the current research provide a strong rationale for the implementation of spatial occlusion goggles as a training tool in football. A significant factor that must be considered when interpreting the improvements experienced in the current research is that no improvement in the performance of one variable occurred as a result of a detrimental impact to another performance variable. For instance, an improvement in response accuracy did not occur 
as a result of an increase in response time. The practical applications for football training is present as the occlusion goggles can be implemented to guide the visual system toward relevant informational sources in environment while improving pass accuracy and speed of the pass. The integration of the spatial occlusion goggles into an in-situ training environment during phase three provides a higher level of transfer to the game. This is due to the experimental design maintaining the critical interaction between performer and environment as well as perception-action coupling.

\section{Disclosure statement}

No potential conflict of interest was reported by the authors.

\section{References}

Abernethy B. 1990. Anticipation in squash: differences in advance cue utilization between expert and novice players. J Sports Sci. 8(1):17-34. doi:10.1080/02640419008732128.

Araújo D, Davids K, Passos P. 2007. Ecological validity, representative design, and correspondence between experimental task constraints and behavioral setting: comment on Rogers, Kadar, and Costall (2005). Ecol Psychol. 19(1):69-78. doi:10.1080/10407410709336951.

Bush M, Barnes C, Archer D, Hogg B, Bradley P. 2015. Evolution of match performance parameters for various playing positions in the English premier league. Hum Mov Sci. 39:1-11. doi:10.1016/j.humov.2014.10.003

Cohen JW. 1988. Statistical power analysis for the behavioral sciences. 2nd ed. Hillsdale (NJ): Lawrence Erlbaum Associates.

Dicks M, Button C, Davids K. 2010. Examination of gaze behaviors under in situ and video simulation task constraints reveals differences in information pickup for perception and action. Attention Percept Psychophys. 72(3):706-720. doi:10.3758/app.72.3.706.

Dicks M, Davids K, Button C. 2009. Representative task designs for the study of perception and action in sport. Int J Sport Psychol. 40(4):506-524.

Dunton A, O' Neill C, Jermyn S, Dawson D, Coughlan E. 2019a. The impact of spatial occlusion goggles on the basketball crossover dribble. Eur J Sports Exercise Sci. 7(1):20-30.

Dunton A, O'Neill C, Coughlan E. 2019b. The impact of a training intervention with spatial occlusion goggles on controlling and passing a football. Sci Med Football. 1-6. doi:10.1080/24733938.2019.1616106

Farrow D, Abernethy B. 2003. Do expertise and the degree of perception-action coupling affect natural anticipatory performance? Perception. 32(9):1127-1139. doi:10.1068/p3323.

Jones C, Miles J. 1978. Use of advance cues in predicting the flight of a lawn tennis ball. J Hum Mov Stud. 4:231-235.
Liu H, Gomez M, Lago-Peñas C, Sampaio J. 2015. Match statistics related to winning in the group stage of 2014 Brazil FIFA World Cup. J Sports Sci. 33 (12):1205-1213. doi:10.1080/02640414.2015.1022578.

Magill RA. 2011. Motor learning and control: concepts and applications. 9th ed. New York: McGraw-Hill.

McGuckian T, Cole M, Chalkley D, Jordet G, Pepping G. 2018a. Visual exploration when surrounded by affordances: frequency of head movements is predictive of response speed. Ecol Psychol. 31(1):30-48. doi:10.1080/10407413.2018.1495548.

McGuckian T, Cole M, Jordet G, Chalkley D, Pepping G. 2018b. Don't turn blind! The relationship between exploration before ball possession and on-ball performance in association football. Front Psychol. 9. doi:10.3389/fpsyg.2018.02520.

Milner AD, Goodale MA. 1995. The visual brain in action. Oxford (England): Oxford University Press.

Müller S, Brenton J, Rosalie S. 2015. Methodological considerations for investigating expert interceptive skill in in situ settings. Am Psychol Assoc. 15:1-13.

Murgia M, Sors F, Muroni A, Santoro I, Prpic V, Galmonte A, Agostini T. 2014. Using perceptual home-training to improve anticipation skills of soccer goalkeepers. Psychol Sport Exerc. 15(6):642-648. doi:10.1016/j. psychsport.2014.07.009.

Nimmerichter A, Weber N, Wirth K, Haller A. 2015. Effects of video-based visual training on decision-making and reactive agility in adolescent football players. Sports. 4(1):1. doi:10.3390/sports4010001.

Oppici L, Panchuk D, Serpiello F, Farrow D. 2017. Long-term practice with domain-specific task constraints influences perceptual skills. Front Psychol. 8(8):1387. doi:10.3389/fpsyg.2017.01387.

Panchuk D, Vickers JN. 2009. Using spatial occlusion to explore the control strategies used in rapid interceptive actions: predictive or prospective control? J Sports Sci. 27(12):1249-1260. doi:10.1080/02640410903 156449.

Pinder R, Davids K, Renshaw I, Araújo D. 2011. Representative learning design and functionality of research and practice in sport. J Sport Exercise Psychol. 33(1):146-155. doi:10.1123/jsep.33.1.146.

Poulter D, Jackson R, Wann J, Berry D. 2005. The effect of learning condition on perceptual anticipation, awareness, and visual search. Hum Mov Sci. 24(3):345-361. doi:10.1016/j.humov.2005.06.005.

Sheppard J, Young W, Doyle T, Sheppard T, Newton R. 2006. An evaluation of a new test of reactive agility and its relationship to sprint speed and change of direction speed. J Sci Med Sport. 9(4):342-349. doi:10.1016/j. jsams.2006.05.019.

Van der Kamp J, Rivas F, van Doorn H, Savelsbergh GJP. 2008. Ventral and dorsal contributions in visual anticipation in fast ball sports. Int J Sport Psychol. 39:100-130.

Vickers JN. 2007. Perception, cognition, and decision training: the quiet eye in action. Champaign (IL): Human Kinetics.

Williams AM, Jackson RC. 2019. Anticipation and decision making in sport. New York (NY): Routledge. 